\title{
LIE GROUP REPRESENTATIONS ON POLYNOMIAL RINGS ${ }^{1}$
}

\author{
BY BERTRAM KOSTANT ${ }^{2}$ \\ Communicated by Raoul Bott, February 1, 1963
}

0 . Introduction. 1. Let $G$ be a group of linear transformations on a finite dimensional real or complex vector space $X$. Assume $X$ is completely reducible as a $G$-module. Let $S$ be the ring of all complexvalued polynomials on $X$, regarded as a $G$-module in the obvious way, and let $J \subseteq S$ be the subring of all $G$-invariant polynomials on $X$.

Now let $J^{+}$be the set of all $f \in J$ having zero constant term and let $H \subseteq S$ be any graded subspace such that $S=J^{+} S+H$ is a $G$-module direct sum. It is then easy to see that

$$
S=J H .
$$

(Under mild assumptions $H$ may be taken to be the set of all $G$ harmonic polynomials on $X$. That is, the set of all $f \in S$ such that $\partial f=0$ for every homogeneous differential operator $\partial$ with constant coefficients, of positive degree, that commutes with $G$.)

One of our main concerns here is the structure of $S$ as a $G$-module. Regard $S$ as a $J$-module with respect to multiplication. Matters would be considerably simplified if $S$ were free as a $J$-module. One shows easily that $S$ is $J$-free if and only if $S=J \otimes H$. This, however, is not always the case. For example $S$ is not $J$-free if $G$ is the two element group $\{I,-I\}$ and $\operatorname{dim} X \geqq 2$. On the other hand one has

Example 1. It is due to Chevalley (see [2]) that if $G$ is a finite group generated by reflections then indeed $S=J \otimes H$. Furthermore the action of $G$ on $H$ is equivalent to the regular representation of $G$.

EXAMPLE 2. $S$ is $J$-free in case $G$ is the full rotation group (with respect to some Euclidean metric on $X$. For convenience assume in this example that $\operatorname{dim} X \geqq 3$ ). Note that the decomposition of a polynomial according to the relation $S=J \otimes H$ is just the so-called "separation of variables" theorem for polynomials. This is so because $J$ is the ring of radial polynomials and $H$ is the space of all harmonic polynomials (in the usual sense).

Now, for any $x \in X$, let $O_{x} \subseteq X$ denote the $G$-orbit of $x$ and let $S\left(O_{x}\right)$ be the ring of all functions on $O_{x}$ defined by restricting $S$ to $O_{x}$. Since $J$ reduces to constants on any orbit it follows that (0.1.1) in-

${ }^{1}$ This research was supported by National Science Foundation grant NSFG19992.

2 The author is an Alfred P. Sloan fellow. 
duces a $G$-module epimorphism

$$
H \rightarrow S\left(O_{x}\right) .
$$

Since our major concern is the case where $X$ is a reductive Lie algebra and $G$ is the adjoint group and since the methods used there belong to algebraic geometry we will assume now that $X$ is complex and that $G$ is algebraic and reductive. All varieties considered are over $C$. If $Y$ has an algebraic structure $R(Y)$ will denote the ring of everywhere defined rational functions in $Y$. Obviously one always has

$$
S\left(O_{x}\right) \subseteq R\left(O_{x}\right)
$$

On the other hand if $G^{x} \subseteq G$ is the isotropy group defined by $x \in X$ then one has a $G$-module isomorphism

$$
R\left(G / G^{x}\right) \rightarrow R\left(O_{x}\right)
$$

The significance of $(0.1 .4)$ is that one knows the $G$-module structure of $R\left(G / G^{x}\right)$ completely by a very simple algebraic Frobenius reciprocity theorem (even though $G^{x}$ may not be reductive). In fact if $V^{\lambda}$ is any irreducible $G$-module with respect to the representation $\nu^{\lambda}$ and $V_{\lambda}$ is the dual module then one has

$$
\text { mult. of } \nu^{\lambda} \text { in } R\left(G / G^{x}\right)=\operatorname{dim} V_{\lambda}^{G^{x}}
$$

where $V_{\lambda}^{G^{x}}$ is the space of vectors in $V_{\lambda}$ fixed under $G^{x}$.

Now in Examples 1 and 2 (assume complexified) the following three optimum situations occur:

(a) $S$ is $J$-free so that $S=J \otimes H$,

(b) the map $H \rightarrow S\left(O_{x}\right)$ is an isomorphism for certain $x \in X$ and for those $x$,

(c) $R\left(O_{x}\right)=S\left(O_{x}\right)$.

But one observes that if in any general case (b) and (c) hold then, clearly, upon combining (0.1.4) and (0.1.5) one gets the $G$-module structure of $H$. If one gets in addition the "graded" $G$-module structure of $H$ and knows the structure of $J$ then one gets the full graded $G$-module structure of $S$ in case (a) also holds.

In Example 2 the conditions (b) and (c) hold for any $x \neq 0$ (even if $(x, x)=0$ ). In fact, classically, one has exploited (b) and (c) for $(x, x)>0$ to solve the Dirichlet problem with the sphere as boundary. That is, if $f$ is any continuous function on the sphere one first expands $f$ as a Fourier development of spherical harmonics $f_{n}$. The sphere is $O_{x} \cap R^{m}$ and the $f_{n}$ are in $R\left(O_{x}\right)$. The equality $R\left(O_{x}\right)=S\left(O_{x}\right)$ and the isomorphism $H \rightarrow S\left(O_{x}\right)$ then yields the extension of $f_{n}$ uniquely as 
harmonic polynomials $h_{n}$ on $X$. But this yields the desired extension of $f$.

In Example 1 the conditions (b) and (c) are satisfied for any "regular" element $x \in X$.

Our first concern in this paper is to give criteria for (a), (b) and (c) to hold in general. Since our interest is in the continuous case we will assume $G$ is connected (and hence a variety). Thus Example 2 rather than Example 1 serves as a model.

Now let $P \subseteq X$ be the cone of common zeros defined by the ideal $J^{+} S$ in $S$. Let $X^{*}$ be the dual space to $X$ and let $P^{*} \subseteq X^{*}$ be defined in a similar way with the roles of $X$ and $X^{*}$ interchanged. As a criterion to establish (a) and more we prove

Proposition 0.1. Assume (1) that $J^{+} S$ is a prime ideal in $S$ and (2) there exists an orbit $O_{e} \subseteq P$ which is dense in $P$. Then $S=J \otimes H$. Furthermore if $G$ is a subgroup of the complex rotation group then $H$ may be taken as the space of all G-harmonic polynomials. Moreover $H$ then coincides with the space spanned by all powers $f^{k}$ where $f \in P^{*}$.

It may be observed that the criterion is satisfied in Example 2.

An element $x \in X$ is called quasi-regular if $P \subseteq \mathrm{Cl}\left(C^{*} \cdot O_{x}\right)$. A criterion to establish (b) is given by

Proposition 0.2. Assume conditions (1) and (2) of Proposition 0.1 are satisfied. Then the G-module epimorphism $H \rightarrow S\left(O_{x}\right)$ is an isomorphism for any quasi-regular element $x \in X$.

It may be observed that in Example 2 every nonzero $x \in X$ is quasi-regular.

From known facts in algebraic geometry one has the following criterion to insure (c).

Proposition 0.3. Let $x \in X$ and assume (1) the closure $\mathrm{Cl}\left(O_{x}\right)$ is a normal variety and $(2) \mathrm{Cl}\left(O_{x}\right)-O_{x}$ has a codimension of at least 2 in $\mathrm{Cl}\left(O_{x}\right)$. Then $R\left(O_{x}\right)=S\left(O_{x}\right)$.

It may be observed that the conditions of Proposition 0.3 are satisfied for every $x \in X$ in Example 2.

Now assume that $X=\mathfrak{g}$ is a complex reductive Lie algebra and $G$ is the adjoint group. Here the structure of $J$ is given by a theorem of Chevalley. This asserts that $J$ is a polynomial ring in $l$ (the rank of $\mathfrak{g}$ ) homogeneous generators $u_{i}, i=1,2, \cdots, l$, with deg $u_{i}=m_{i}+1$ where the $m_{i}$ are the exponents of $\mathfrak{g}$. 
Now one knows that here $P$ is the set of all nilpotent elements of $\mathfrak{g}$ [13, Theorem 9.1]. But then by [13, Corollary 5.5], $P$ does contain a dense orbit $O_{e}$, namely, the set of all principal nilpotent elements in g. Thus to apply Propositions 0.1 and 0.2 one must prove that $J^{+} S$ is a prime ideal.

If $n=\operatorname{dim} \mathfrak{g}$ (all dimensions are over $C$ ) then one sees easily that $n-l$ is the maximal dimension of any orbit. Let $\mathfrak{r}=\left\{x \in \mathfrak{g} \mid \operatorname{dim} O_{x}\right.$ $=n-l\}$. Any regular element $x \in \mathfrak{g}$ belongs to $\mathfrak{r}$. But also $e \in \mathfrak{r}$ for any principal nilpotent element $e$. These in fact are extreme cases.

Proposition 0.4. Let $x \in \mathfrak{g}$ be arbitrary. Write (uniquely) $x=y+z$ where $y$ is semi-simple, $z$ is nilpotent and $[y, z]=0$. Let $\mathrm{g}^{y}$ be the centralizer of $y$ in $\mathfrak{g}$ so that $\mathfrak{g}^{y}$ is a reductive Lie algebra and $z \in \mathfrak{g}^{y}$. Then $x \in \mathfrak{r}$ if and only if $\boldsymbol{z}$ is principal nilpotent in $\mathfrak{g}^{\nu}$.

Let $x \in \mathfrak{g}$. Consider the values $\left(d u_{i}\right)_{x}$ of the $l$ differential forms $d u_{i}, i=1,2, \cdots, l$, at $x$. It is known that these covectors are linearly independent whenever $x$ is regular. (One recalls that the product of the positive roots is an $l \times l$ minor of a suitable $n \times l$ matrix determined by the $d u_{i}$.) But to prove the primeness of the ideal $J^{+} S$ one needs to know that these covectors are linearly independent if $x$ is a principal nilpotent element. This fact is contained in

Theorem 0.1. Let $x \in \mathfrak{g}$. Then the $\left(d u_{i}\right)_{x}$ are linearly independent if and only if $x \in \mathfrak{r}$.

Proposition 0.1 may now be applied.

Theorem 0.2. One has $S=J \otimes H$ where $H$ is the space of all $G$ harmonic polynomials on $\mathfrak{g}$. Furthermore $H$ coincides with the space of all polynomials spanned by all powers of "nilpotent" linear functionals.

Since Theorem 0.1 shows also that $P$ is a complete intersection the decomposition $S=J \otimes H$ when combined with [15, Proposition 5, $\S 78]$ gives, in the notation of $\mathrm{FAC}$, all the sheaf cohomology groups $H^{j}(\boldsymbol{P}, \mathcal{O}(m))$ where $\boldsymbol{P}$ is the projective variety defined by $P$.

Added in proof. Another application of the primeness of $J^{+} S$ in algebraic geometry is

TheOREM 0.3 (Added in proof). The intersection multiplicity of $P$, at the origin, with any Cartan subalgebra is w, where w is the order of the Weyl group.

Next, Proposition 0.2 is put into effect for all orbits of maximal dimension by

THEOREM 0.4. The set $\mathfrak{r}$ coincides with the set of all quasi-regular ele- 
ments in $\mathfrak{g}$. (Thus $H$ and $S\left(O_{x}\right)$ are isomorphic as G-modules for any $x \in \mathfrak{r}$.)

As a consequence of Theorems 0.2 and 0.4 one shows that not only is the ideal $J^{+} S$ prime in $S$ but $J_{1} S$ is prime for any prime ideal $J_{1} \subseteq J$. Furthermore one gets the following characterization of all the invariant prime ideals in $S$ which are generated by elements of $J$.

TheOREM 0.5. Let $I \subseteq S$ be any $G$-invariant prime ideal. Let $\mathfrak{u} \subseteq \mathfrak{g}$ be the affine variety of zeros of $I$. Then $I$ is of the form $I=J_{1} S$ for $J_{1} a$ prime ideal in $J$ if and only if $u \cap \mathfrak{r}$ is not empty.

Since $R\left(O_{x}\right)=S\left(O_{x}\right)$ in case $O_{x}$ is closed and since $O_{x}$ is closed if $x$ is regular one gets the $G$-module structure of $H$ by applying Theorem 0.3 and (0.1.5) for $x$ regular. Thus if $D$ denotes the set of dominant integral forms corresponding to a Cartan subgroup $A$, so that $D$ indexes all the irreducible representations of $G$ as highest weights, then one has

$$
\text { mult. of } \nu^{\lambda} \text { in } H=l_{\lambda}
$$

where $l_{\lambda}=\operatorname{dim} V_{\lambda}^{A}$ is the multiplicity of the zero weight of $\nu_{\lambda}$.

In order to determine the $G$-module structure of $S^{k}$, the space of homogeneous polynomials on $\mathfrak{g}$ of degree $k$, one must know more than (0.1.6). In fact using the relation $S=J \otimes H$ what one wants is the multiplicity of $\nu^{\lambda}$ in $H^{j}=S^{i} \cap H$ for any $\lambda$ and $j$. As it turns out, for this, one needs $R\left(O_{e}\right)=S\left(O_{e}\right)$ where $e$ is a principal nilpotent element. To show the latter using Proposition 0.3 it is enough to show that $P$ is a normal variety and $P-O_{e}$ has a codimension of at least 2 in $P$.

Let $\mathcal{O}_{\mathfrak{r}}$ be a set of all orbits of maximal dimension $(n-l)$. The set $\mathcal{O}_{\mathfrak{r}}$ may be parameterized by $\boldsymbol{C}^{l}$ in the following way. Let

$$
u: \mathfrak{g} \rightarrow \mathrm{C}^{\imath}
$$

be the morphism given by putting $u(x)=\left(u_{1}(x), \cdots, u_{l}(x)\right)$ for any $x \in \mathfrak{g}$. Since $u$ reduces to a constant on any orbit it induces a map

$$
\eta_{\mathfrak{r}}: \mathcal{O}_{\mathfrak{r}} \rightarrow C^{l} .
$$

One has

THEOREM 0.6. $\eta_{\mathfrak{r}}$ is a bijection.

Thus to each $\xi \in C^{l}$ there exists a unique orbit, $O(\xi)$ of dimension $n-l$ which correspond to $\xi$ under $\eta_{\mathfrak{r}}$. Now let $P(\xi)=u^{-1}(\xi)$ for any $\xi \in C^{l}$ so that 


$$
\mathfrak{g}=\bigcup_{\xi \in C^{l}} P(\xi)
$$

is a disjoint union. Note that $P(\xi)=P$ and $O(\xi)=O_{e}$ if $\xi$ is the origin of $\mathbf{C}^{l}$. One proves

ThEOREM 0.7. For any $\xi \in C^{l}$ one has

$$
P(\xi)=\mathrm{Cl}(O(\xi))
$$

so that $P(\xi)$ is a variety of dimension $n-l$. Moreover $P(\xi)$ is a complete intersection and $O(\xi)$ coincides with the set of simple points on $P(\xi)$. Finally $P(\xi)$ is a finite union of orbits so that $\mathrm{Cl}\left(O_{x}\right)$ is a finite union of orbits for any $x \in \mathfrak{g}$.

Since $P(\xi)$ is a complete intersection and since its singular locus is the complement (a finite union of orbits) of $O(\xi)$ in $P(\xi)$ one would get the normality of $P(\xi)$ by a theorem of Seidenberg if one knew the dimension of the other orbits in $P(\xi)$ were at most $n-l-2$.

Now it is well known that $\operatorname{dim} O_{x}$ is even (and hence $\operatorname{dim}_{R} O_{x}$ is a multiple of 4) for any semi-simple element $x \in \mathfrak{g}$. Less known is the following proposition observed independently by the author, Borel, and (most simply proved by) Kirillov.

Proposition 0.5. The dimension of $O_{x}$ is even for any $x \in \mathfrak{g}$.

Combining Theorem 0.6 and Proposition 0.5 one obtains

Theorem 0.8. Let $\xi \in C^{l}$ be arbitrary. Then $P(\xi)$ is a normal variety and the codimension of $P(\xi)-O(\xi)$ in $P(\xi)$ is at least 2 .

Applying Proposition 0.3 one then has

Theorem 0.9. Let $x \in \mathfrak{r}$. Then $R\left(O_{x}\right)=S\left(O_{x}\right)$. (This implies that all $R\left(O_{x}\right)$ for $x \in \mathfrak{r}$ are isomorphic as $G$-modules; even though they are not in general isomorphic as rings.) Let $\xi=u(x)$. Then $R\left(O_{x}\right)\left(=R\left(G / G^{x}\right)\right)$ is an affine algebra (even though $O_{x}$ is not necessarily an affine variety) and $P(\xi)$ is the variety of all maximal ideals of $R\left(O_{x}\right)$. Thus the embedding of $G / G^{x}$ in $g$ as $O_{x}$ is special in that any morphism of $G / G^{x}$ (or $O_{x}$ ) into any affine variety extends uniquely to a morphism of $P(\xi)=\mathrm{Cl}\left(O_{x}\right)$ into the variety. (In particular this holds for $O_{e}$ and $\mathrm{Cl}\left(O_{e}\right)=P$.) Finally (using (0.1.5) and the equality $R\left(O_{x}\right)=S\left(O_{x}\right)$ ) one has, for any $\lambda \in D$

$$
\operatorname{dim} V_{\lambda}^{G^{x}}=l_{\lambda}
$$

so that the left side of (0.1.7) is independent of $x \in \mathfrak{r}$.

Now let $e_{-}, x_{0}, e$ be a principal $S$-triple (that is, a "canonical" basis 
of a principal three dimensional simple Lie subalgebra). In particular then $e$ is a principal nilpotent element. Used heavily in the theorems above is the result of [13] which asserts that $g^{e}$ is $l$-dimensional and has a basis $z_{i}, i=1,2, \cdots, l$, such that

$$
\left[x_{0}, z_{i}\right]=m_{i} z_{i}
$$

where, we recall, the $m_{i}$ are the exponents of $\mathfrak{g}$. But now since $\mathfrak{g}^{e}=\mathfrak{g}^{G^{e}}$ (because $\mathfrak{g}^{e}$ is commutative) and since (0.1.7) holds for $x=e$ this suggests a generalization of the notion of exponent. Let $V$ be any finite dimensional $G$-module with respect to a representation $\nu$. If $l_{\nu}$ is the multiplicity of the zero weight of $\nu$ then by (0.1.7) one has $\operatorname{dim} V^{G^{\circ}}$ $=l_{\nu}$. It follows therefore that there exists a unique nondecreasing sequence of non-negative integers $m_{i}(\nu), i=1,2, \cdots, l_{\nu}$, such that one has

$$
\nu\left(x_{0}\right) z_{i}=m_{i}(\nu) z_{i}
$$

for a basis $z_{i}$ of $V^{G^{\theta}}$. If $\nu$ is the adjoint representation the $m_{i}(\nu)$ are the usual exponents. If $\nu=\nu^{\lambda}$ we will write $m_{i}(\lambda)$ for $m_{i}\left(\nu^{\lambda}\right)$ and note (because the highest weight has multiplicity one) that

$$
m_{j}(\lambda)=o(\lambda) \quad \text { for } j=l_{\lambda}
$$

where $o(\lambda)$ is the sum of the coefficients of $\lambda$ relative to the simple roots and that this highest value occurs with multiplicity one among the generalized exponents $m_{i}(\lambda)$. (This specializes to the familiar relation $m_{l}=o(\psi)$ when $g$ is simple and $\psi$ is the highest root.)

The following theorem now gives the $G$-module structure of $H^{j}$ and hence $S^{k}$ for any $j$ and $k$.

TheORem 0.10. Let $\lambda \in D$ be arbitrary and let $H(\lambda)$ be the set of $G$ harmonic polynomials which transform under $G$ according to $\nu^{\lambda}$. Let (by (0.1.6)) $H(\lambda)=\sum_{j=1}^{\lambda_{\lambda}} H_{j}(\lambda)$ be a decomposition into irreducible components so that $H_{j}(\lambda) \subseteq H^{n_{i}}$ where $n_{j}, j=1,2, \cdots, l_{\lambda}$, is a nondecreasing sequence of integers. Then $n_{j}=m_{j}(\lambda)$ for all $j$. In particular then $k=o(\lambda)$ is the highest degree $k$ such that $\nu^{\lambda}$ occurs in $H^{k}$. Moreover it occurs with multiplicity one for this value of $k$.

Assume for convenience that $\mathfrak{g}$ is simple and let $\psi \in D$ be the highest root. Let $x_{i}, i=1,2, \cdots, n$, be a basis of $\mathfrak{g}$. If the $u_{j} \in J$ are chosen properly one sees that $\partial u_{j} / \partial x_{i}, i=1,2, \cdots, n$, is a basis of $H_{j}(\psi)$. One notes then that Theorem 0.10 is a generalization of the result in [13] given by (0.1.8).

H. S. Coxeter observed and A. J. Coleman proved in [4] that if $W$ is the Weyl group and $\sigma \in W$ is the Coxeter-Killing transformation 
then the eigenvalues of $\sigma$ operating on the Cartan subalgebra are $e^{2 \pi i m_{j} / s}, j=1,2, \cdots, l$, where $s$ is order of $\sigma$. Now more generally $W$ operates on the zero weight space of $V^{\lambda}$ for any $\lambda \in D$ according (say) to some representation $\pi^{\lambda}$ of $W$. As a generalization of the CoxeterColeman theorem one now has

TheOREM 0.11. For any $\lambda \in D$ the eigenvalues of $\pi^{\lambda}(\sigma)$ are $e^{2 \pi i m_{j}(\lambda) / s}$, $j=1,2, \cdots, l_{\lambda}$.

0.2. By applying the Birkhoff-Witt theorem the results above carry over from $S$ to $U$, the universal enveloping of $\mathfrak{g}$ ( $U$ is obviously a $G$ module in a natural way).

THEOREM 0.12. Let $U$ be the universal enveloping algebra over $\mathfrak{g}$ and let $Z \subseteq U$ be the center of $U$. Then $U$ is free as a $Z$-module (under multiplication). In fact

$$
U=Z \otimes E
$$

where $E$ is the subspace (and G-submodule) of $U$ spanned by all powers $x^{k}$ for all nilpotent elements $x \in \mathfrak{g}$. Moreover $E$ is equivalent to $H$ as $a$ $G$-module so that every irreducible representation of $G$ occurs with finite multiplicity in $E$ (in fact $\nu^{\lambda}$ occurs $l_{\lambda}$ times in $E$ for any $\lambda \in D$ ).

Let $V$ be a finite dimensional irreducible $U$-module so that one has a $G$-module algebra epimorphism

$$
\rho: U \rightarrow \text { End } V \text {. }
$$

Since $\rho(Z)$ reduce to the scalars it follows from $(0.2 .1)$ that $\rho(E)$ $=$ End $V$. Now let $Y$ be any subspace of $U$. If $Y$ is one-dimensional then it is due to Harish-Chandra that there exists an irreducible $U$ module $V$ such that $\rho$ is faithful on $Y$. This is not true in general if $\operatorname{dim} Y \geqq 2$. However it is true if $Y \subseteq E$.

THEOREM 0.13. Let $Y \subseteq E$ be any finite dimensional subspace. Then there exists an irreducible $U$-module $V$ such that $\rho$ is faithful on $Y$.

I would like to express my thanks to C. Chevalley, M. Rosenlicht and A. Seidenberg for helpful conversation about questions in algebraic geometry. In particular to Seidenberg for making me aware of his criterion for normality and to Chevalley for simplifying my proof of the primeness of $J+S$.

\section{REFERENCES}

1. A. Borel and Harish-Chandra, Arithmetic subgroups of algebraic groups, Ann. of Math. (2) 75 (1962), 485-535. 
2. C. Chevalley, Invariants of finite groups generated by reflections, Amer. J. Math. 77 (1955), 778-782.

3. - Fondements de las géométric algébrique, Course notes at the Institut Henri Poincaré, Paris, 1958.

4. A. J. Coleman, The Betti numbers of the simple Lie groups, Canad. J. Math. 10 (1958), 349-356.

5. E. B. Dynkin, Semi-simple subalgebras of semi-simple Lie algebras, Amer. Math. Soc. Transl. (2) 6 (1957), 111-244.

6. F. Gantmacher, Canonical representation of automorphisms of a complex semisimple Lie group, Mat. Sb. 47 (1939), 104-146.

7. Harish-Chandra, On a lemma of F. Bruhat, J. Math. Pures Appl. (9) 35 (1956), 203-210.

8. - On representations of Lie algebras, Ann. of Math. (2) 50 (1949), 900-915.

9. G. Hochschild and G. D. Mostow, Representations and representative functions on Lie groups. III, Ann. of Math. (2) 70 (1959), 85-100.

10. S. Helgason, Some results in variant theory, Bull. Amer. Math. Soc. 68 (1962), 367-371.

11. N. Jacobson, Completely reducible Lie algebras of linear transformations, Proc. Amer. Math. Soc. 2 (1951), 105-133.

12. B. Kostant, A formula for the multiplicity of a weight, Trans. Amer. Math. Soc. 93 (1959), 53-73.

13. - The principal three-dimensional subgroup and the Bette numbers of a complex simple Lie group, Amer. J. Math. 81 (1959), 973-1032.

14. M. Rosenlicht, On quotient varieties and the affine embedding of certain homogeneous spaces, Trans. Amer. Math. Soc. 101 (1961), 211-223.

15. J. P. Serre, Faisceaux algebriques cohérent, Ann. of Math. (2) 61 (1955), 197278.

16. A. Seidenberg, The hyperplane sections of normal varieties, Trans. Amer. Math. Soc. 64 (1950), 357-386.

17. R. Steinberg, Invariants of finite reflection groups, Canad. J. Math. 12 (1960), 616-618.

18. O. Zariski and P. Samuel, Commutative algebra, Vol. I, Van Nostrand, Princeton, N. J., 1958.

19. - Commutative algebra, Vol. II, Van Nostrand, Princeton, N. J., 1960.

Massachusetts Institute of Technology 